\title{
Eight items of the ruminative response scale are sufficient to measure weekly within-person variation in rumination
}

\author{
Annette Brose ${ }^{1,2,3}$ (D) Rudi De Raedt ${ }^{4}$ - Marie-Anne Vanderhasselt ${ }^{4,5}$ \\ Published online: 18 July 2020 \\ (C) The Author(s) 2020
}

\begin{abstract}
The Ruminative Response Scale (RRS) is commonly used to measure people's general tendency to ruminate. In this study, we explored whether only few items from the RRS can be used to capture within-person variation in rumination in intensive longitudinal studies. Such a short RRS version would allow, for example, monitoring the development of rumination during clinical interventions. We measured rumination on five occasions, with at least one week in between. We used multilevel analyses to analyze the data at the within- and between-person level. Using only eight RRS items, we successfully modeled a reflective self-regulation and depressive brooding factor, similar to the two subfacets of rumination as distinguished by Treynor et al. (2003). We also established convergent validity of depressive brooding at the within- and between-person level of analysis and convergent validity of reflection at the between-person level. We thus introduced a short form of the RRS that captures within-person variation in depressive brooding and reflection well. The short RRS is readily applicable in studies on withinperson variation or change in rumination.
\end{abstract}

Keywords Rumination $\cdot$ Within-person variation $\cdot$ Diary $\cdot$ Ruminative response scales $\cdot$ Measurement

Rumination involves repetitively and passively focusing on symptoms of distress, as well as on the causes and consequences of these symptoms (Nolen-Hoeksema et al. 2008). Although people who ruminate often do so with the belief that they are solving a problem, a habitual tendency to ruminate is associated with increased sadness, distress and anxiety. Rumination is a well-known cognitive vulnerability mechanism for depression (Nolen-Hoeksema 2000; NolenHoeksema and Morrow 1991; Robinson and Alloy 2003). In other words, if ruminative thinking is not actively targeted in treatment, significant research has shown it will result in

Annette Brose

annette.brose@hu-berlin.de

1 Institute of Psychology, Humboldt-Universität zu Berlin, Unter den Linden 6, 10099 Berlin, Germany

2 Max Planck Institute for Human Development, Lentzeallee 94, 14195 Berlin, Germany

3 KU Leuven, Oude Markt 13, 3000 Leuven, Belgium

4 Department of Experimental Clinical and Health Psychology, Ghent University, 9000 Ghent, Belgium

5 Department of Head and Skin, Unit of Psychiatry and Medical Psychology, Ghent University, Ghent, Belgium slower symptom reduction, and can lead to a poorer response to therapy. Therefore, it is important to measure rumination at regular intervals across time in order to elucidate changes in rumination and gain insight into developments in the course of individuals' treatment over time.

The most widely used measure of rumination is the Ruminative Response Scale (RRS; cf. Treynor et al. 2003). The scale measures the tendency to use ruminative thinking when being in a negative mood, and is derived from the Response Styles Theory by Nolen-Hoeksema (1987). This theory implies a bidirectional, within-person link between rumination and distress in the sense that rumination increases distress and distress increases rumination (Nolen-Hoeksema and Morrow 1993; Lyubomirsky and Tkach 2004; see also Moberly and Watkins 2008). Two distinct styles of rumination, with distinct functional properties and consequences, have been reported. Brooding entails self-criticism and negative evaluations of one's current status in comparison to some standard. Reflection is related to more active problem solving - thoughts are used to overcome some difficulty.

Even though the RRS is a well-validated questionnaire, with acceptable between-person reliabilities of the brooding and reflection subscales $(\alpha=.77$ and .72 , respectively, Treynor et al. 2003), the whole scale is rather long (22 items) and is most suitable in the assessment of a general tendency to 
ruminate when in a negative mood. Put differently, this measure has not been adapted to measure within-person variation regarding the tendency to ruminate across a relatively brief period of time (e.g., days or weeks). It is therefore not yet appropriate for repeated, frequent use during the course, for example, of a psychotherapeutic intervention. Also, to our knowledge, no other measure of within-person variation in rumination exists that captures brooding and reflection reliably, albeit various investigations of rumination at the within-person level. For example, rumination was measured with one or two items only in various experience sampling studies (e.g., Brans et al. 2013; Moberly and Watkins 2008), and it was also measured with six items from the RRS in one study (Genet and Siemer 2012). However, none of these studies provided information on the within-person reliability of the scale, nor did it explicitly touch the issue of validity. An exception is the study by Newman and Nezlek (2019). They measured rumination and reflection with three items each, the items stemming from the trait Rumination-Reflection Questionnaire (Trapnell \& Campbell, 1999). They could report acceptable reliability only for the rumination subscale (within-person alpha $=.78$ ), but for the reflection subscale; within-person alpha was .59, even after the attempt to improve reliability by deleting one item from the scale.

Given this background, the purpose of this study was to develop a short form of the RRS that might be a worthy candidate for repeated measurements of rumination. Such a short form should illuminate within-person variation in rumination that might be reversible, for example when it occurs as reaction to stressful events (Vanderhasselt et al. 2016), or that might show some general trend across time, for example a decline in case of successful interventions (Vanderhasselt et al. 2015).

Our development of a short form of the RRS was guided by three criteria: The set of items to measure within-person variation in rumination should (1) be a brief instrument; (2) have acceptable psychometric properties; (3) ideally capture the two established subscales of rumination. The three criteria were based on the following rationales: (1) It is common to work with brief instruments in studies with multiple repeated assessments (e.g., diary studies, clinical interventions) because study participants usually fill out the same items repeatedly. The latter places relatively much burden on participants. Hence, researchers need to make sure that compliance remains high. Keeping measurement instruments short, especially when assessing multiple constructs repeatedly, is one recommendation to achieve this (Shiffman et al. 2008). Ideally, however, one works with at least three items per construct because a minimum of three items is necessary to identify a common factor. (2) Acceptable psychometric properties of measurement instruments are an essential prerequisite in descriptive and inferential research, and this applies to measurement instruments capturing between-person variation as well as those capturing within-person variation across time. Guidelines for approaching good psychometric properties at the withinperson level have, however, only been established rather recently (Brose et al. 2020; Nezlek 2017), and we thus specifically highlight this criterion. (3) A within-person measure of rumination should capture the two validated facets of rumination, brooding and reflection, because they were previously shown to have different predictive validity (e.g., Treynor et al. 2003). Only if both aspects are kept for within-person research can future work determine whether brooding and reflection are also distinguishable subfacets of rumination with distinct validity at the within-person level.

For scale development, we used data from a study in which rumination was measured with the RRS on five occasions in a stressful context, with at least one week inbetween. To reiterate, we aimed at a selection of items from the RRS that have the best psychometric properties to capture within-person variation in the tendency to ruminate. Participants were asked to complete a modified version of the RRS across different occasions. Modification of the standard RRS was done such that the weekly measure captured rumination across that last week. A non-clinical student sample was selected in which rumination was measured in and out of a stressful period. Research in nonclinical samples has shown that rumination emerges in the context of stress and affective distress and prolongs a stressor-related affective reaction. For example, rumination keeps attention on some stressor and on related negative feelings and thereby impedes more active problem solving and prolongs negative affect (Lyubomirsky et al. 1999). Moreover, peoples' affective reactions to stressors are stronger when they strongly ruminate (Genet and Siemer 2012), and rumination partly mediates effects of stressors on affective distress within individuals across time (Moberly and Watkins 2008). Following these notions, we also examined the convergent validity of the two presumed subscales by establishing relationships with candidate variables, specifically depressive symptoms and negative affect.

Our main analytical approach was multilevel confirmatory factor analysis (Muthen 1994). Furthermore, to test the convergent validity at the within-person level, we used multilevel regression analysis that revealed within-person co-variation between rumination and other time varying covariates (e.g., depressive symptoms).

\section{Method}

This study is part of a larger project investigating information processes associated with different forms of emotion regulation (see Vanderhasselt et al. 2016). 


\section{Participants and Procedures}

A total of ninety-two students of Ghent University (72 females, 20 males) with a mean age of 20.27 years $(S D=$ 2.04) participated in this study. After receiving a complete verbal description of the study, participants provided written informed consent (protocol approved by the local ethics committee of Ghent University). At the end, they all received a financial reward for participation. Of the longitudinal study, six occasions are relevant to this manuscript. Individual difference characteristics (e.g., trait level rumination, depressive symptoms, and negative affect) were administered at the first occasion (T1). The next five sessions (T2 to T6) measured time-varying aspects of different variables (e.g., weekly rumination, weekly depressive symptoms).

\section{Measures}

Rumination, Standard Version of the RRS, T1 To measure between-person differences in rumination, we used the Dutch translation of the Ruminative Response Scale (RRS; Nolen-Hoeksema and Morrow 1991; Treynor et al. 2003; Dutch translation by Raes and Hermans 2007, RRSNL; see also Schoofs et al. 2010). This Dutch self-report questionnaire consists of 26 questions to which participants respond using a 4-point Likert scale (i.e. $1=$ almost never, $2=$ sometimes, $3=$ often, $4=$ most of the time). Questions deal with general behaviors and cognitions that people are aware of when they feel depressed. Prior work on the RRS identified two separate subscales, brooding and reflection, that were differentially related to psychological distress at the betweenperson level (Treynor et al. 2003; see also Takano and Tanno 2009). Reflection consists of five questions, assessing the degree to which individuals engage in cognitive selfregulation to reduce negative mood. More maladaptive ways of rumination, i.e. brooding, include five questions assessing the extent to which individuals passively focus on the reasons for their distress (Treynor et al., 2003). Scores reflecting between-person differences in reflection and brooding were computed in accordance with these subscales.

Rumination, Weekly Version of the RRS, T2 - T6 Within-person variation in rumination was also measured with the 26item Dutch version of the RRS, using the same 4-point answering scale. Yet, participants were asked to report on rumination over the last week. The development of two brief subscales from this long measurement instrument is described below.

Rumination, Standard Version of the CERQ, T1 Between-person variation in rumination was also measured with a subscale of the Cognitive Emotion Regulation Questionnaire (CERQ; Garnefski et al. 2001). The subscale consists of four items of which the mean was used for analyses. Participants were asked to indicate the extent to which they generally use a specific cognitive strategy. The answering scale is a 5-point Likert scale ranging from 1 ([almost] never) to 4 ([almost] all the time).

Rumination, Weekly Version of the CERQ, T2 - T6 The CERQ was adapted to also measure within-person variation. The participants were asked to provide information on the extent to which they experienced the thoughts formulated in the CERQ across the last week. The response scale was equivalent to that of the standard version. We used the mean across the four items for the analyses of convergent validity at the withinperson level.

Depressive Symptoms, Standard Version, $\mathrm{T} 1$ The Beck Depression Inventory II (BDI-II; Beck et al. 1996; Dutch translation by Van der Does 2002) was administered to screen for between-person differences in depressive symptoms and dysphoria. The BDI consists of 21 multiple-choice items and measures the presence and severity of symptoms of depression. In addition to an aggregated score across all BDI items, we aggregated a subset of items that specifically reflect negative feelings (i.e. feeling sad/unhappy, bored, guilty, irritated; referred to as BDI-negative feelings). This score was analyzed as another indicator of the convergent validity of the new measure. Obviously, this score overlaps with the other BDI score. Yet, it is of particular interest when studying rumination because rumination essentially implies ongoing thoughts on negative feelings.

Depressive Symptoms, Weekly Version, T2 - T6 The BDI was also adapted to measure weekly variation in depressive symptoms. Participants were asked to think of the last week when reporting on the symptoms of depression. In the analyses of convergent validity at the within-person level, we analyzed the mean across all items as well as the mean of items that reflect negative feelings.

Negative Affect, Standard Version of the PANAS, T1 Betweenperson variation in negative affect was measured with the Positive and Negative Affect Schedule (PANAS; Watson et al. 1988). The negative affect subscale consists of 10 items of which the mean was used for analyses. The response scale is a 6-point Likert scale ranging from 1 (not so much) to 5 (a lot).

\section{Analytical Approach}

Item Selection We used multilevel confirmatory factor analysis (MCFA; ran in Mplus 8.3) to establish the most suitable set of items for measuring within-person variation in brooding and reflection. The purpose of MCFA is to simultaneously 
estimate a within-person and a between-person factor model in a confirmatory fashion (i.e., on the basis of theoretical considerations). In MCFA, the total covariance is separated into within- and between-person components. The within-person covariance matrix is then estimated from the pooled covariance matrices for each person (Muthen 1994), and the between-person covariance matrix is estimated for the estimated means. The emerging within-person structure reflects the structure of within-person variation, and the emerging between-person structure reflects the structure of betweenperson variation.

To judge the goodness of fit of the assumed two-factor structure of within-person and between-person variation in rumination (with the two correlated factors reflection and brooding), we followed the recommendations by $\mathrm{Hu}$ and Bentler (1999). They proposed that the following values for several fit indices indicate a good fit of the model to the data: a Comparative Fit Index (CFI) close to .95 (the higher the better), a standardized root mean squared residual (SRMR) close to .08 (the lower the better), and a root mean squared error of approximation (RMSEA) of .06 (the lower the better). All analyses were carried out using maximum likelihood estimation with robust standard errors (MLR; Mplus default for MCFA).

The within-person reliabilities of the brooding and reflection subscales were determined from one-dimensional measurement models in MCFA (Geldhof et al. 2014). We chose to run two separate models for brooding and reflection because we were working with a relatively small number of observations (Geldhof et al. 2014), specifically a small number of observations within Level-2 units. We report omega as reliability coefficient. Omega is computed from the factor loadings in MCFA. It is advantageous to alpha as the the loadings do not underlie the assumption of essential tau equivalence, thereby allowing for heterogeneous item-construct relations that are commonly found. We did not do a priori power analyses in this study, but simulation studies by Geldhof et al. (2014) seem to suggest that our sample size of 92 participants and a total of 457 occasions should be sufficiently large for our examination of the psychometric properties of a new measure for within-person variation in rumination. In their examination of the performance of level-specific reliability estimates under varying conditions, Geldhof et al. (2014) concluded that within- and between-level reliability estimates are generally unbiased, unless several conditions occur (when "within-level reliability is low and there are relatively few, small clusters" [p. 89], and "when item ICCs were low, especially when the between-cluster reliability was also low and when there were few observations per cluster" [p. 89]). The number of clusters and size of clusters (i.e., sample sizes) in these conditions were smaller than those in our study. We therefore imply that we likely obtained relatively unbiased reliability estimates at both levels.

Convergent Validity To test the convergent validity of the emerging factors (brooding and reflection, based on the MCFA) at the within-person level, we used multilevel modelling. Brooding and reflection were the time-varying criterion variables in these models. Time-varying predictor variables were rumination (measured with the CERQ), depressive symptoms (measured with the BDI), and BDI-negative feelings, all measured at $\mathrm{T} 2$ to $\mathrm{T} 6$ and modelled as predictors in separate models. The emerging Level-1 multilevel regression coefficients reflect the within-person co-variation of brooding / reflection and each of the predictor variables across time. In particular, we modelled both fixed and random effects at Level-1, whereas the former indicate the average withinperson co-variation and the latter individuals' deviations from the average coefficients. Predictor variables were personmean centered prior to analyses. We provide unstandardized

Table 1 Descriptive Statistics

\begin{tabular}{llr}
\hline & $M$ & $S D$ \\
\hline Brooding, standard RRS & 2.04 & 1.06 \\
Reflection, standard RRS & 1.89 & 0.71 \\
Rumination, standard CERQ & 3.05 & 1.06 \\
Depressive symptoms, standard BDI & 0.29 & 0.30 \\
Negative feelings, standard BDI & 0.22 & 0.34 \\
Negative affect, standard PANAS & 1.94 & 0.56 \\
Depressive brooding, short-version RRS, average across occasions & 1.65 & 0.59 \\
Reflective self-regulation, short-version RRS, average across occasions & 1.53 & 0.42 \\
Rumination, CERQ, average across occasions & 2.54 & 0.79 \\
Depressive symptoms, BDI, average across occasions & 0.35 & 0.30 \\
Negative feelings, BDI, average across occasions & 0.33 & 0.29 \\
\hline
\end{tabular}

Note. RRS $=$ Ruminative Response Scale CERQ $=$ Cognitive Emotion Regulation Questionnaire, $\mathrm{BDI}=$ Beck Depression Inventory; PANAS = Positive and Negative Affect Schedule 
Table 2 Items of the Short-Version RRS and their Within-Person Factor Loadings from two Separate Measurement Models

Std. factor loading

Depressive brooding

\begin{tabular}{llll}
1 & I think about how only I feel this way. & .51 & .58 \\
2 & I wonder why I have these problems and others don't. & .43 & .59 \\
3 & I think about how sad I feel. & .77 & .70 \\
4 & I think about my failures. & .54 & .64 \\
5 & I try to understand my depressed feelings. & .47 & .61 \\
Reflective self-regulation & .49 \\
6 & I write down what I am thinking and analyze it. & .67 & .55 \\
7 & I go out alone and think about why I am feeling this way. \\
8 & I go somewhere on my own to think about my feelings. & .80 \\
\hline
\end{tabular}

Note. Std. $=$ standardized; ICC $=$ intraclass correlation

multilevel regression coefficients as well as information on the amount of variance in rumination / reflection that is explained by predictors (i.e., the pseudo- $\mathrm{R}^{2}$ statistic).

To test the convergent validity of the emerging factors (rumination and reflection) at the between-person level, we correlated the latent variables (brooding and reflection) with other indicators of between-person differences (i.e., brooding and reflection scores from the standard RRS, rumination scores from the standard CERQ, depressive symptoms scores from the standard BDI, negative feelings scores from the standard BDI, and negative affect scores from the standard PANAS).

\section{Results}

\section{Descriptive Statistics}

Descriptive statistics are reported in Table 1. Please note that the means and standard deviations reported for the withinperson measures (lower part of the table) are based on aggregates per individual across $\mathrm{T} 2$ to $\mathrm{T} 6$.

\section{Development of the Short-Version RRS}

To establish the most suitable set of items we departed from the Dutch version of the RRS. To reiterate briefly, the criteria for scale development were to establish a brief measure that is suited for studies with repeated assessments, has acceptable psychometric properties, is in accordance with a standard definition of rumination, and captures distinguishable aspects of rumination (brooding and reflection).

\section{Measurement Models}

We started with two separate measurement models for the two subscales of the RRS, reflection and brooding. For the reflection subscale, we selected those three items from the RRS as reported by Treynor et al. (2003) that do not have a reference to negative feelings (Table 2). To avoid confusion with the original reflection scale, we renamed the scale into reflective self-regulation. The three items were modelled as indicators of a latent reflection factor at the within- and between-person level using MCFA. All items had sufficient within-person variance, as indicated by the ICCs (reported in Table 2). The model fit of this multilevel one-factor model was good, RMSEA $=<.001, \mathrm{CFI}=1.0, \mathrm{SRMR}_{\text {within }}=.001$ and $\mathrm{SRMR}_{\text {between }}=.004$. The emerging factor loadings are presented in Table 2. Next, we estimated the reliability of the reflective self-regulation subscale (i.e., composite reliability / omega), the estimates being omega $a_{\text {within }}=.71$, omega $_{\text {between }}=.94$.

For the brooding subscale, we started out with items from this subscale as reported by Treynor et al. (2003). The items were modelled as indicators of a latent brooding factor at the within- and between-person level using MCFA. None of the combinations of items (combinations of three to five items of the Treynor items) resulted in a reliable subscale of brooding at the within-person level (i.e., composite reliability was $<.60)$. To nevertheless obtain a reliable within-person subscale for brooding, we switched from our confirmatory to an exploratory approach. Specifically, we used multilevel exploratory factor analysis ${ }^{1}$ on a selection of twelve items from the RRS, with the goal to find relatively homogenous items as indicated by factor loadings. The selection of the twelve items was guided by a defining feature of rumination - a focus of thoughts on symptoms of distress (Nolen-Hoeksema et al. 2008). The emerging set of five items with the highest loadings on the brooding factor is presented in Table 2 .

\footnotetext{
$\overline{1}$ Given our focus on a reliable measure for within-person variation, we modelled one within-person factor and an unrestricted between-person covariance matrix.
} 
In a final step, these items were tested as indicators of brooding in a confirmatory way, again using MCFA. All items had sufficient within-person variance, as indicated by the ICCs (Table 2). The model fit of this MCFA was good, RMSEA $=.04$, CFI $=.99$, SRMR $_{\text {within }}=.03$ and $\mathrm{SRMR}_{\text {between }}=.03$. The reliabilities of this set of items ${ }^{2}$ were omega $_{\text {within }}=.67$, and omega $a_{\text {between }}=.94$. Given the more negative content of this brooding subscale in comparison to that by Treynor et al. (2003), we call this factor depressive brooding.

Together, these analyses provided two subscales, depressive brooding and reflective self-regulation, with good model fit for the final measurement models.

\section{Modelling the 2-Dimensional Structure of Brooding and Reflection}

Next, we tested whether rumination as measured with the new depressive brooding and reflective self-regulation subscales is indeed a two-dimensional construct at the within- and the between-person level of analysis. We tested the model fit of a two-dimensional model, including the two subscales, again using MCFA. The result is presented in Fig. 1. The model fit was acceptable, RMSEA $=.06, \mathrm{CFI}=.94, \mathrm{SRMR}_{\text {within }}=.05$ and $\mathrm{SRMR}_{\text {between }}=.06$, and the correlations between factors indicated commonality and divergence between the two latent dimensions, $r_{\text {within }}=.51, r_{\text {between }}=.67$. We compared this model to a model in which factor correlations were fixed to 1 (which would indicate indistinctness of the factors), using $\chi^{2}$ difference testing. The result suggested that both factors should be kept, and thus, the existence of two distinguishable subscales.

\section{Convergent Validity}

\section{Within-Person Level}

The results of the analyses of convergent validity at the within-person level are reported in Table 3. Given this study's specific interest in the coefficients that are informative on convergent validity, we only present the Level-1 coefficients representing within-person associations. Moreover, we report the amount of variance that the predictor variables explain in brooding and reflection (i.e., the pseudo- $\mathrm{R}^{2}$ statistic).

The new depressive brooding subscale significantly covaried with rumination as measured with the CERQ, with depressive symptoms, and with BDI-negative feelings. That is, occasions on which study participants had particularly high

\footnotetext{
${ }^{2}$ We also ran other one-factor MCFAs with less than five indicators of a common brooding factor (from those five items with highest loadings in the exploratory analysis). The within-person reliabilities of these subsets decreased to below .65. Thus, we kept the items as presented in Table 5 as indicators of depressive brooding.
}

.51

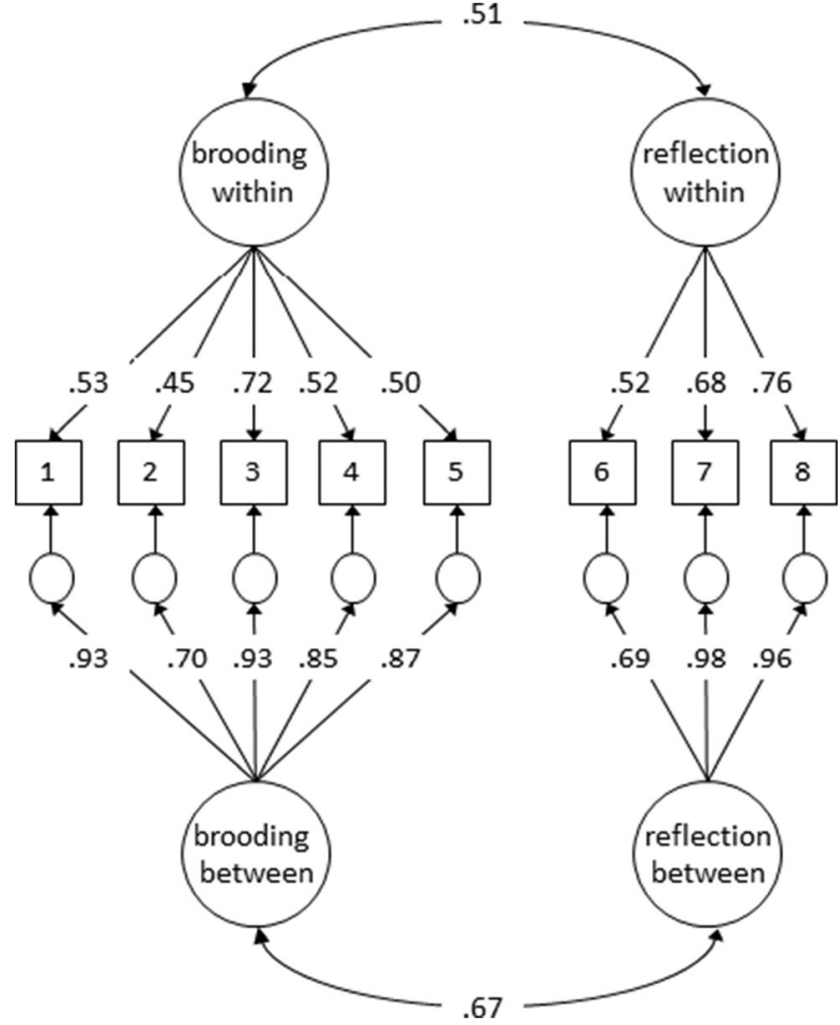

Fig. 1 Schematic Figure of the Multilevel Confirmatory Factor Model Used to Examine the Short RRS Version Note. Brooding and reflection refer to depressive brooding and reflective self-regulation of the shortversion RRS; loadings are standardized; factor loadings of Items 7 and 8 were constrained to equality at the between-person level.

levels of depressive brooding were occasions on which they also had high levels of rumination (CERQ), depressive symptoms and BDI-negative feelings. The amount of variance in within-person depressive brooding that was explained by these predictors ranged between 25 and $27 \%$.

The new reflective self-regulation subscale significantly co-varied with CERQ- rumination at the within-person level. Yet, the amount of variance that CERQ-rumination explained in within-person reflection is small (1\%). Moreover, reflection was neither significantly associated with depressive symptoms nor with BDI-negative feelings at the within-person level. The amount of variance in within-person reflection that is explained by depressive symptoms and BDI-negative feelings is $9 \%$ in both cases.

\section{Between-Person Level}

The results for the analyses of convergent validity at the between-person level are reported in Table 4. All correlations between the new depressive brooding and reflective selfregulation subscale and the indicators of convergent validity were significant. In more detail, the correlations between the new depressive brooding subscale and the standard measures of rumination (i.e., the standard brooding subscale of the RRS; 
Table 3 Convergent Validity of the Short-Version RRS Subscales at the Within-Person Level

\begin{tabular}{llll}
\hline & Rumination, CERQ & Depressive symptoms, BDI & BDI-negative feelings \\
\hline $\begin{array}{l}\text { Depressive brooding } \\
\text { estimate (SE) }\end{array}$ & $0.05(0.01)^{*}$ & $0.03(0.01)^{*}$ & $0.19(0.07)^{*}$ \\
$\begin{array}{c}\text { explained wp-variance } \\
\text { Reflective self-regulation }\end{array}$ & $25 \%$ & $27 \%$ & $27 \%$ \\
$\begin{array}{l}\text { estimate (SE) } \\
\text { explained wp-variance }\end{array}$ & $0.02(0.01)^{*}$ & $0.01(.004)$ & $0.09(0.06)$ \\
\hline
\end{tabular}

Note. $* p<.05$; CERQ = Cognitive Emotion Regulation Questionnaire, BDI = Beck Depression Inventory the rumination subscale of the CERQ) were .54 and .51 , respectively. Moreover, the correlations between the new brooding subscale and depressive symptoms and BDInegative feelings were .64 and .54 , respectively. The size of the correlations of the brooding subscale and negative affect was .49 .

The correlation between the reflective self-regulation subscale and the standard reflection subscale of the RRS was also high, $r=.60$. The correlations between this subscale and the other measures were moderate, with the range of the correlations being .24 to .33 . The smallest correlations were found between reflection and the standard brooding subscale as well as with BDI-negative feelings.

\section{Discussion}

We successfully selected eight items of the RRS that are wellsuited for the study of within-person, weekly variation in rumination, in particular, depressive brooding and reflective self-regulation. The subscales are in accordance with a standard definition of rumination (Nolen-Hoeksema et al. 2008), and the analyses of convergent validity revealed strong relationships with standard measures of brooding, reflection, and rumination at the between-person level. Furthermore, there was convincing evidence for convergent validity of the depressive brooding subscale at the within-person level.
In more detail, the reliabilities (internal consistencies) of the new subscales were .67 and .71, with good model fit of both measurement models, meaning that the chosen items capture the two underlying latent dimensions of rumination well. In the case of the brooding subscale, within-person reliability was a bit lower than the well-known standard of .70 (Cronbach 1951), but moderate in accordance with Shrout (1998). Regarding the analyses of convergent validity, the depressive brooding subscale shared between 25 and $27 \%$ within-person variance with rumination and depressive symptoms as measured with the CERQ and the BDI. The reflective self-regulation subscale, instead, was on average not related to these variables, or, in the case of the CERQ, shared only very little within-person variance. That is, at the within-person level, the reflection subscale is on average rather independent from CERQ-rumination and depressive symptoms. This indicates that the content of the two subscales is well distinguishable at the within-person level, despite the positive within-person correlation between the two subscales. Consequently, the two subscales should allow distinguishing between more maladaptive versus adaptive aspects of rumination at the within-person level, just as the brooding and reflection subscales by Treynor et al. (2003) seem to reflect more and less adaptive aspects of rumination at the between-person level. If used in the context of some intervention, for example, one may expect divergent trajectories of these two variables: a decrease of depressive brooding and an increase in reflective self-regulation.
Table 4 Convergent Validity of the Short RRS Subscales at the Between-Person Level

\begin{tabular}{lllllll}
\hline & $\begin{array}{l}\text { Brooding, } \\
\text { RRS, T1 }\end{array}$ & $\begin{array}{l}\text { Reflection, } \\
\text { RRS, T1 }\end{array}$ & $\begin{array}{l}\text { Rumination, } \\
\text { CERQ, T1 }\end{array}$ & $\begin{array}{l}\text { Depressive } \\
\text { symptoms, } \\
\text { BDI, T1 }\end{array}$ & $\begin{array}{l}\text { BDI- } \\
\text { negative } \\
\text { feelings, T1 }\end{array}$ & $\begin{array}{l}\text { Negative } \\
\text { affect, } \\
\text { PANAS, T1 }\end{array}$ \\
\hline $\begin{array}{c}\text { Depressive } \\
\text { brooding } \\
\begin{array}{c}\text { Reflective } \\
\text { self-- } \\
\text { regulation }\end{array}\end{array}$ & $.54^{*}$ & $.46^{*}$ & $.51^{*}$ & $.64^{*}$ & $.54^{*}$ & $.49^{*}$ \\
\hline
\end{tabular}

Note. $* p<.05$; RRS $=$ Ruminative Response Scale; estimates are correlations of the brooding and reflection factor at the between-person level with the indicators of convergent validity (first row of the table); CERQ = Cognitive Emotion Regulation Questionnaire, BDI = Beck Depression Inventory; PANAS = Positive and Negative Affect Schedule 
This study's findings at the between-person level also speak for the distinctness of depressive brooding and reflective selfregulation. They imply that individuals with high average levels of depressive brooding across weeks were those who also had high levels of rumination and depressive symptoms according to standard measures at $\mathrm{T} 1$. Individuals with high average levels of reflection across weeks had particularly high levels of reflection at $\mathrm{T} 1$. These correlations furthermore speak for convergent validity at the between-person level.

We consider the selection of items of the RRS for within-person research as an important step in research on dynamic aspects of rumination, which may be important for clinical purposes. A ruminative thinking style is an important mechanism in relation to the onset of dysfunctional attitudes, based on its association with negative attentional biases, sustained negative mood states, and increased vulnerability for depression (for a review, see Smith and Alloy 2008). Data from a prior study in our lab revealed that rumination predicts the activation of dysfunctional attitudes and that this relation is fully mediated by the experience of depressive symptoms (Vanderhasselt and De Raedt 2012). Hence, clinical interventions should aim to reduce ruminative thinking as it is an important underlying vulnerability mechanism that sets depressive symptoms and dysfunctional attitudes in motion. A valid and reliable measurement of change in rumination is therefore necessary to investigate whether the intervention is successful. This is also important to map cognitive and emotional processes throughout the intervention, as these two variables likely co-vary with rumination within individuals across time.

This study also comes with some drawbacks. First, the time scale underlying variation in the RRS items is variation across weeks. Ideally, a measure of within-person variation would be validated on multiple time scales. That is, we know from this study that the selected RRS items capture weekly variations well. Whether this finding would generalize to daily variations, for example, remains to be investigated. Nevertheless, the weekly time scale is a timescale that might be of relevance in intervention studies because therapy sessions are often scheduled weekly. The monitoring of rumination during the course of interventions seems very feasible with the selected items from the RRS. A second limitation of this study is that it did not include a specific indicator of convergent validity of reflection at the within-person level. We know from the analyses that the reflection items of the RRS are not related to rumination and depressive symptoms as measured with a weekly version of the CERQ and BDI, respectively. This finding is evidence for the distinctiveness of brooding and reflection at the within-person level (see above), and we would not necessarily have expected significant correlations between the reflection subscales and the other within-person measures. Thirdly, the depressive brooding items differ from the brooding subscale by Treynor et al. (2003) in an important way: they have more overlap of depressive symptoms (Items 3 and 4, Table 2). To highlight this difference, we termed this subscale depressive brooding rather than brooding. The high between-person correlation of this subscale with the standard brooding subscale by Treynor and colleagues makes us confident, however, that these subscales are sufficiently related. Related to this issue, we obtained the items for the depressive brooding subscale using exploratory factor analysis. That is, we switched from a confirmatory to an exploratory approach and back. Ideally, one would do so using data from independent samples. We could not do this in the absence of a second study with similar measures. Given this limitation, the establishment of our new measure would ideally be followed up with new data.

In sum, the increasing amount of research that tackles psychological phenomena at the within-person level calls for measurement instruments whose psychometric properties are also established at the within-person level (Brose et al. 2020; Nezlek 2017). Here, we introduced a short-form of the RRS that captures within-person variation in rumination well, in particular in terms of variation in depressive brooding and reflective self-regulation. With eight items, the new measure is short enough to be administered multiple times in intensive longitudinal studies. The short-version RRS is readily applicable in studies on weekly variations or change in rumination (e.g., intervention studies that monitor rumination on the timescale of weekly treatments). The use of this and other established measures of within-person variation will warrant that research at the within-person level of psychological phenomena becomes more comparable across studies.

Funding Open Access funding provided by Projekt DEAL. This study was funded by a grant by the German Research Foundation [Deutsche Forschungsgemeinschaft, DFG], BR 3782/3-1; a grant BOFSTA2017002501 for research at Ghent University (BOFSTA2017002501) and a grant for a Concerted Research Action of Ghent University (Grant BOF16/GOA/017).

\section{Compliance with Ethical Standards}

Conflict of Interests All authors declare that they have no conflict of interest.

Ethical Approval All procedures performed in studies involving human participants were in accordance with the ethical standards of the institutional and/or national research committee and with the 1964 Helsinki declaration and its later amendments or comparable ethical standards. This article does not contain any studies with animals performed by any of the authors.

Informed Consent Informed consent was obtained from all individual participants included in the study.

Open Access This article is licensed under a Creative Commons Attribution 4.0 International License, which permits use, sharing, adaptation, distribution and reproduction in any medium or format, as long as 
you give appropriate credit to the original author(s) and the source, provide a link to the Creative Commons licence, and indicate if changes were made. The images or other third party material in this article are included in the article's Creative Commons licence, unless indicated otherwise in a credit line to the material. If material is not included in the article's Creative Commons licence and your intended use is not permitted by statutory regulation or exceeds the permitted use, you will need to obtain permission directly from the copyright holder. To view a copy of this licence, visit http://creativecommons.org/licenses/by/4.0/.

\section{References}

Beck, A, T., Steer, R, A., \& Brown, G, K. (1996). Manual for the Beck depression inventory (2nd ed.). San Antonio, TX: The Psychological Corporation.

Brans, K., Koval, P., Verduyn, P., Lim, Y. L., \& Kuppens, P. (2013). The regulation of negative and positive affect in daily life. Emotion, 13(5), 926-939. https://doi.org/10.1037/a0032400.

Brose, A., Schmiedek, F., Gerstorf, D., \& Voelkle, M. C. (2020). The measurement of within-person affect variation. Emotion, 20(4), 677-699. https://doi.org/10.1037/emo0000583.

Cronbach, L. J. (1951). Coefficient alpha and the internal structure of tests. Psychometrika, 16, 297-334. https://doi.org/10.1007/ BF02310555.

Garnefski, N., Kraaij, V., \& Spinhoven, P. (2001). Negative life events, cognitive emotion regulation and emotional problems. Personality and Individual Differences, 30, 1311-1327.

Geldhof, G. J., Preacher, K. J., \& Zyphur, M. J. (2014). Reliability estimation in a multilevel confirmatory factor analysis framework. Psychological Methods, 19, 72-91.

Genet, J. J., \& Siemer, M. (2012). Rumination moderates the effects of daily events on negative mood: Results from a diary study. Emotion, $12,1329-1339$.

Hu, L.-T., \& Bentler, P. M. (1999). Cutoff criteria for fit indexes in covariance structure analysis: Conventional criteria versus new alternatives. Structural Equation Modeling, 6, 1-55. https://doi.org/ 10.1080/10705519909540118.

Lyubomirsky, S., \& Tkach, C. (2004). The consequences of dysphoric rumination. In C. Papageorgiou \& A. Wells (Eds.), Depressive rumination (pp. 21-43). West Sussex: Wiley.

Lyubomirsky, S., Tucker, K. L., Caldwell, N. D., \& Berg, K. (1999). Why ruminators are poor problem solvers: Clues from the phenomenology of dysphoric rumination. Journal of Personality and Social Psychology, 77, 1041-1060.

Moberly, N. J., \& Watkins, E. R. (2008). Ruminative self-focus and negative affect: An experience sampling study. Journal of Abnormal Psychology, 117, 314-323.

Muthen, B. O. (1994). Multilevel covariance structure analysis. Sociological Methods \& Research, 22, 376-398. https://doi.org/ 10.1177/0049124194022003006.

Newman, D. B., \& Nezlek, J. B. (2019). Private self-consciousness in daily life: Relationships between rumination and reflection and well-being, and meaning in daily life. Personality and Individual Differences, 136, 184-189. https://doi.org/10.1016/j.paid.2017.06.039.

Nezlek, J. B. (2017). A practical guide to understanding reliability in studies of within-person variability. Journal of Research in Personality, 69, 149-155. https://doi.org/10.1016/j.jrp.2016.06. 020.

Nolen-Hoeksema, S. (1987). Sex differences in unipolar depression: Evidence and theory. Psychological Bulletin, 101, 259-282.

Nolen-Hoeksema, S. (2000). The role of rumination in depressive disorders and mixed anxiety/depressive symptoms. Journal of Abnormal Psychology, 109, 504-511.
Nolen-Hoeksema, S., \& Morrow, J. (1991). A prospective study of depression and posttraumatic stress symptoms after a natural disaster: The 1989 Loma Prieta earthquake. Journal of Personality and Social Psychology, 61, 115-121.

Nolen-Hoeksema, S., \& Morrow, J. (1993). Effects of rumination and distraction on naturally occurring depressed mood. Cognition \& Emotion, 7, 561-570. https://doi.org/10.1080/02699939308409206.

Nolen-Hoeksema, S., Wisco, B. E., \& Lyubomirsky, S. (2008). Rethinking rumination. Perspectives on Psychological Science, 3, 400-424.

Raes, F., \& Hermans, D. (2007). The revised version of the Dutch ruminative response scale. Unpublished instrument.

Robinson, M. S., \& Alloy, L. B. (2003). Negative cognitive styles and stress-reactive rumination interact to predict depression: A prospective study. Cognitive Therapy and Research, 27, 275-292.

Schoofs, H., Hermans, D., \& Raes, F. (2010). Brooding and reflection as subtypes of rumination: Evidence from confirmatory factor analysis in nonclinical samples using the Dutch ruminative response scale. Journal of Psychopathology and Behavioral Assessment, 32, 609617.

Shiffman, S., Stone, A. A., \& Hufford, M. R. (2008). Ecological momentary assessment. Annual Review of Clinical Psychology, 4, 1-32.

Shrout, P. E. (1998). Measurement reliability and agreement in psychiatry. Statistical Methods in Medical Research, 7, 301-317. https:// doi.org/10.1191/096228098672090967

Smith, J. M., \& Alloy, L. B. (2008). A roadmap to rumination: A review of the definition, assessment, and conceptualization of this multifaceted construct. Clinical Psychology Review, 29, 116-128.

Takano, K., \& Tanno, Y. (2009). Self-rumination, self-reflection, and depression: Self-rumination counteracts the adaptive effect of selfreflection. Behavior Research and Therapy, 47, 260-264. https:// doi.org/10.1016/j.brat.2008.12.008.

Trapnell, P. D., \& Campbell, J. D. (1999). Private self-consciousness and the five-factor model of personality: Distinguishing rumination from reflection. Journal of Personality and Social Psychology, 76(2), 284-304. https://doi.org/10.1037/0022-3514.76.2.284

Treynor, W., Gonzalez, R., \& Nolen-Hoeksema, S. (2003). Rumination reconsidered: A psychometric analysis. Cognitive Therapy and Research, 27, 247-259.

Van der Does, A, J, W. (2002). De Nederlandse versie van de Beck depression inventory -Tweede Editie. [the Dutch version of the Beck depression inventory - Second edition]. Lisse: Swets \& Zeitlinger.

Vanderhasselt, M.-A., \& De Raedt, R. (2012). How ruminative thinking styles lead to dysfunctional cognitions: Evidence from a mediation model. Journal of Behavior Therapy and Experimental Psychiatry, 43, 910-914.

Vanderhasselt, M. A., De Raedt, R., Namur, V., Lotufo, P. A., Bensenor, I. M., Boggio, P. S., \& Brunoni, A. R. (2015). Transcranial electric stimulation and neurocognitive training in clinically depressed patients: A pilot study of the effects on rumination. Progress in NeuroPsychopharmacology \& Biological Psychiatry, 3(57), 93-99. https://doi.org/10.1016/j.pnpbp.2014.09.015.

Vanderhasselt, M.-A., Brose, A., Koster, E. H. W., \& de Raedt, R. (2016). Co-variation between stressful events and rumination predicts depressive symptoms: An eighteen months prospective design in undergraduates. Behaviour Research and Therapy, 87, 128-133.

Watson, D., Clark, A. L., \& Tellegen, A. (1988). Development and validation of brief measures of positive and negative affect - the PANAS scales. Journal of Personality and Social Psychology, 54, 1063-1070. https://doi.org/10.1037//0022-3514.54.6.1063.

Publisher's Note Springer Nature remains neutral with regard to jurisdictional claims in published maps and institutional affiliations. 